\title{
THERMAL CONVECTION OF MAGNETO COMPRESSIBLE COUPLE- STRESS FLUID SATURATED IN A POROUS MEDIUM WITH HALL CURRENT
}

\author{
C.B. MEHTA* \\ Department of Mathematics \\ Distt Shimla (H.P), INDIA \\ E-mail: chanderbmehta@gmail.com \\ M. SINGH \\ Department of Mathematics \\ Govt. Post Graduate College Seema (Rohru) \\ Distt Shimla (H.P), INDIA \\ S. KUMAR \\ Department of Mathematics \\ St. Bede's College Shimla \\ Distt Shimla (H.P), INDIA
}

Centre of Excellence Govt. Degree College Sanjauli

\begin{abstract}
An investigation is made on the effect of Hall currents on thermal instability of a compressible couple-stress fluid in the presence of a horizontal magnetic field saturated in a porous medium. The analysis is carried out within the framework of the linear stability theory and normal mode technique. A dispersion relation governing the effects of viscoelasticity, Hall currents, compressibility, magnetic field and porous medium is derived. For the stationary convection a couple-stress fluid behaves like an ordinary Newtonian fluid due to the vanishing of the viscoelastic parameter. Compressibility, the magnetic filed and couple-stress parameter have stabilizing effects on the system whereas Hall currents and medium permeability have a destabilizing effect on the system, but in the absence of Hall current couple-stress has a destabilizing effect on the system. It has been observed that oscillatory modes are introduced due to the presence of viscoelasticity, magnetic field porous medium and Hall currents which were non- existent in their absence.
\end{abstract}

Key words: couple-stress fluid, horizontal magnetic field, compressibility, porous medium, Hall currents.

\section{Introduction}

Theoretical and experimental results on thermal convection in a fluid layer, in the absence and presence of magnetic field and rotation, have been given by Chandrasekhar [1]. Magnetohydrodynamics (MHD) finds vital applications in MHD generators, MHD flow-meters and pumps for pumping liquid metals in metallurgy, geophysics, MHD couples and bearings, and physiological processes such as the magnetic therapy. The growing importance of the use of non-Newtonian fluids in modern technology and industries has led various researchers to study diverse flow problems related to several non - Newtonian fluids. One such fluid that has attracted attention of research workers during the four decades is the couple-stress fluid. The theory of couple-stress fluids initiated by Stokes [2] is a generalization of the classical theory of viscous fluids, which allows the presence of couple-stresses and body couples in the fluid medium. The concept of

\footnotetext{
* To whom correspondence should be addressed
} 
couple-stresses arises due to the way in which mechanical interactions in the fluid medium are modeled. In this theory the rotational field is defined in terms of the velocity field itself and the rotation vector equals half the curl of the velocity vector. The stress tensor here is no longer symmetric. An excellent introduction to this theory is available in the monograph of Stokes [3].

One of the applications of the couple-stress fluid is its use in the study of the mechanisms of lubrication of synovial joints which has become the object of scientific research. A human joint is a dynamically loaded bearing which has an articular cartilage as the bearing and synovial fluid as the lubricant. When a fluid film is generated, squeeze - film action is capable of providing considerable protection to the cartilage surface. The shoulder, hip, knee and ankle joints are the loaded bearing synovial joints of the human body and these joints have a low friction coefficient and negligible wear. Normal synovial fluid is a viscous, non - Newtonian fluid and is generally clear or yellowish. The synovial fluid has been modeled as a couple-stress fluid in human joints by Walicki and Walicka [4].

Hall currents are effects whereby a conductor carrying an electric current perpendicular to an applied magnetic field develops a voltage gradient which is transverse to both the current and the magnetic field. It was discovered by Hall in 1879 while he was working on his doctoral degree at Johns Hopkins University at Baltimore, Maryland. Hall effects have again become an active area of research with the discovery of the quantized Hall effect by Klaus von Klitzing for which he was awarded the Nobel Prize of physics in 1985. In ionized gases (plasmas), where the magnetic field is very strong and affects the electrical conductivity, cannot be Hall currents. Recently, instability in either a couple-stress fluid layer or couple- stress fluid saturated porous layer heated from below has been investigated including the external constraints such as the magnetic field.

A layer of a couple-stress fluid saturating a porous medium heated from below in the presence of rotation was studied by Sunil et al. [5], and the condition for the onset of convection was obtained. In another study, Sharma et al. [6] discussed the effect of Hall currents and finite Larmor radius on thermosolutal instability of rotating plasma and established the destabilizing influence of Hall currents. Chandra [7] observed a contradiction between the theory and experiment for the onset of convection in fluids heated from below. He performed the experiment in an air layer and found that the instability depended on the depth of the layer.

For compressible fluids, the equations governing the system become quite complicated. Spiegel et al. [8] simplified the set of equations governing the flow of compressible fluids assuming that the depth of the fluid layer is much smaller than the scale height as defined by them and motions of infinitesimal amplitude are considered. Under these assumptions, the flow equations for compressible fluids are the same as for incompressible fluids except that the static temperature gradient $\beta$ is replaced by its excess over the adiabatic $\beta-\left(g / C_{p}\right) ; C_{p}$ being specific heat of the fluid at constant pressure. Sharma et al. [9] studied the onset of convection in a couple-stress fluid saturated porous layer in the presence of rotation and magnetic field. Malashety and Kollur [10] investigated the double-diffusive convection in a horizontal couple-stress fluid saturated anisotropic porous layer. Singh [11] studied Hall current effect on thermosolutal instability in a viscoelastic fluid saturated porous layer. He observed that the magnetic field and the stable solute gradient are found to have a stabilizing effect, whereas Hall currents and medium permeability have destabilizing effects on the system.

Jaimal et al. [12] studied thermal convection in a couple-stress fluid in the presence of a magnetic field with Hall currents and observed that couple-stress parameter has dual character in the presence of hall currents. Singh and Dixit [13] have studied Hall effects on thermal instability of a compressible couple-stress fluid in the presence of suspended particles. Singh and Kumar [14] studied magneto and rotatory thermosolutal convection in a couple-stress fluid saturated porous layer in case of an incompressible fluid and in another study Singh and Mehta [15] studied in case of compressible fluids observed that couple-stress fluids have a dual character while the magnetic field has a stabilizing effect on the system. Singh and Kumar [16] studied thermosolutal convection in a couple-stress fluid in uniform vertical magnetic field and observed that the couple-stress parameter has a stabilizing effect on the system. Krishna and Neeraja [17] studied Hall effects on an MHD flow of a couple-stress fluid in a parallel plate channel bounded by a porous 
bed on the lower half in the presence of an inclined magnetic field. Rudraiah et al. [18] investigated linear stability of an electro-hydrodynamic purely conducting couple-stress fluid flowing through a porous channel.

During the survey it has been noticed that Hall effects are completely neglected in the studies of couple-stress fluids. Keeping in mind the importance of couple-stress fluids, convection in the fluid layer heated from below, magnetic field and Hall current effects, the present paper attempts to study the effect of Hall current on thermal instability of a couple-stress fluid in the presence of a horizontal magnetic field flowing through a porous channel.

\section{Mathematical formulation of the problem}

Consider an infinite layer of an compressible finitely conducting (both electrically and thermally) couple-stress fluid through a porous medium, confined between two horizontal planes situated at $z=0$ and $z=1$, acted upon by a uniform horizontal magnetic field $H(H, 0,0)$, gravity field $g(0,0,-g)$. The fluid layer is heated from below such that a steady adverse temperature gradient $\beta=\frac{T_{0}-T_{1}}{d}$, where $T_{0}$ and $T_{1}$ are the constant temperatures of the lower and upper boundaries with $T_{0}>T_{1}$ are maintained.

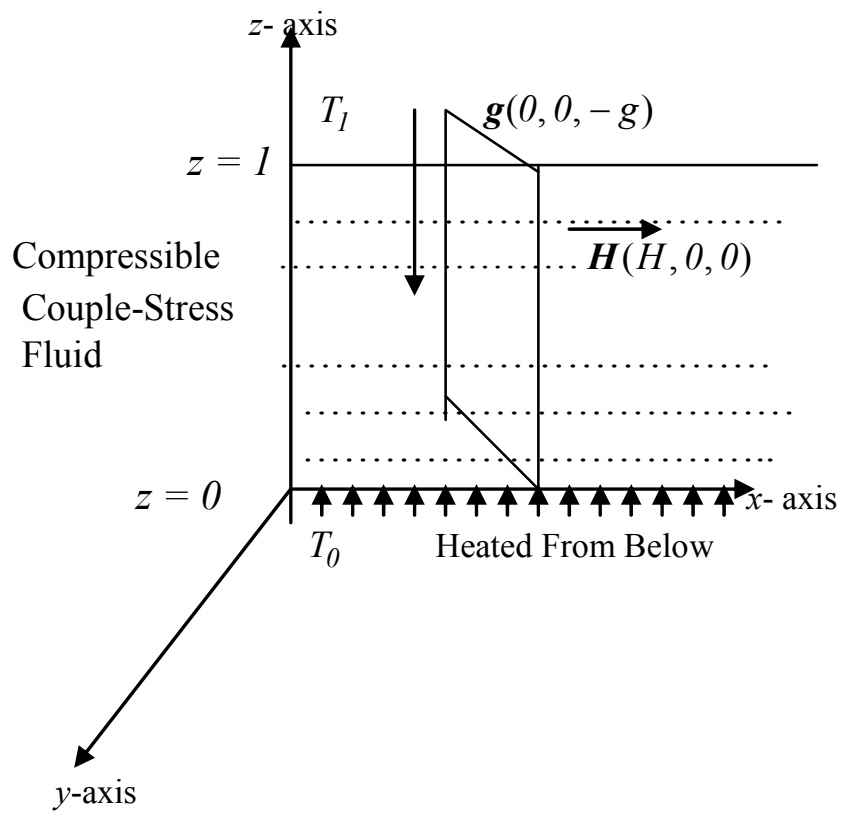

Fig.1. Geometrical configuration.

The hydromagnetic equations (Stokes [3], Chandrasekhar [1]) relevant to the physical model are

$$
\begin{aligned}
& \frac{1}{\in}\left[\frac{\partial q}{\partial t}+\frac{1}{\epsilon}(q . \nabla) q\right]=-\frac{1}{\rho_{0}} \nabla p+g\left(1+\frac{\delta \rho}{\rho_{0}}\right)-\frac{1}{k_{l}}\left(v-\frac{\mu^{\prime}}{\rho_{0}} \nabla^{2}\right) q+\frac{\mu_{e}}{4 \pi \rho_{0}}(\nabla \times H) \times H, \\
& \nabla . q=0 \\
& E \frac{\partial T}{\partial t}+(q \cdot \nabla) T=k \nabla^{2} T,
\end{aligned}
$$




$$
\begin{aligned}
& \nabla . H=0 \\
& \in \frac{d H}{d t}=(H . \nabla) q+\in \eta \nabla^{2} H-\frac{c \in}{4 \pi N_{e}} \nabla \times[(\nabla \times H) \times H]
\end{aligned}
$$

where $\frac{d}{d t}=\frac{\partial}{\partial t}+\epsilon^{-1} q . \nabla$, stands for the convective derivative.

$$
\begin{aligned}
& \rho=\rho_{0}\left[1-\alpha\left(T-T_{0}\right)\right], \\
& E=\in+(1-\in) \frac{\rho_{s} c_{s}}{\rho_{0} c_{v}}, \quad q(0,0,0), \quad T=-\beta z+T_{0}, \quad \rho_{0}=\rho_{0}(1+\alpha \beta z) .
\end{aligned}
$$

In the undisturbed state, let the fluid be at rest. Constant temperatures are maintained in the fluid and a constant horizontal magnetic field is applied, therefore, the steady state solution is given by $q=(0,0,0)$, $H=(H, 0,0), T=T(z), \rho=\rho(z), p=p(z)$.

Let $q(u, v, w), h\left(h_{x}, h_{y}, h_{z}\right), \delta \rho, \delta p$ and $\theta$ are respectively the perturbations in the fluid velocity, magnetic field, density, pressure and temperature and are functions of space as well as time. Then linearized hydromagnetic perturbation equations for couple-stress fluids through porous medium and changed in density $\delta \rho$ caused mainly by the perturbation $\theta$ in temperature are

$$
\begin{aligned}
& \delta \rho=-\alpha \rho_{0} \theta \\
& \frac{1}{\epsilon} \frac{\partial q}{\partial t}=-\frac{1}{\rho_{0}}(\nabla . \delta p)-g \alpha \theta-\frac{1}{k_{1}}\left(v-\frac{\mu^{\prime}}{\rho_{0}} \nabla^{2}\right) q+\frac{\mu_{e}}{4 \pi \rho_{0}}(\nabla \times h) \times H, \\
& \nabla \cdot q=0, \\
& E \frac{\partial \theta}{\partial t}=\left(\beta-\frac{g}{c_{p}}\right) w+k \nabla^{2} \theta, \\
& \in \frac{\partial h}{\partial t}=(H . \nabla) q+\in \eta \nabla^{2} h-\frac{c \in}{4 \pi N_{e}} \nabla \times[(\nabla \times h) \times H], \\
& \nabla . h=0 .
\end{aligned}
$$

\section{Dispersion relation} form

Analyzing the perturbations into normal modes, we assume that the perturbation quantities are of the

$$
\left[w, h_{z}, \theta, \varsigma, \xi\right]=[W(z), K(z), \Theta(z), Z(z), X(z)] \exp \left[i k_{x} x+i k_{y} y+n t\right]
$$

where $k_{x}$ and $k_{y}$ are the wave numbers in $x$ and $y$ directions, respectively, $k=\left(k_{x}^{2}+k_{y}^{2}\right)^{1 / 2}$ is the resultant wave number of propagation and $n$ is the frequency of any arbitrary disturbance which is, in general, a complex constant. 
$\varsigma=\frac{\partial v}{\partial x}-\frac{\partial u}{\partial y}, \xi=\frac{\partial h_{y}}{\partial x}-\frac{\partial h_{x}}{\partial y}$ are the $z$-components of the vorticity and current density, respectively. We eliminate the physical quantities after using the non-dimensional parameters $\sigma=\frac{n d^{2}}{\mathrm{v}}, p_{1}=\frac{\mathrm{v}}{k}, p_{l}=\frac{k_{1}}{d^{2}}, F=\frac{\mu / \rho_{0} d^{2}}{\mathrm{v}}, D=\frac{d}{d z}, G=\frac{c_{p} \beta}{g}$, using Eqs (2.8) - (3.1), we have

$$
\begin{aligned}
& \left(D^{2}-a^{2}\right)\left[\frac{\sigma}{\epsilon}+\frac{1}{p_{l}}-\frac{F}{p_{l}}\left(D^{2}-a^{2}\right)\right] W-\frac{i k_{x} \mu_{e} H d^{2}}{4 \pi \rho_{0} \mathrm{v}}\left(D^{2}-a^{2}\right) K+\frac{g \alpha d^{2} a^{2}}{\mathrm{v}} \Theta=0, \\
& {\left[\frac{\sigma}{\epsilon}+\frac{1}{p_{l}}-\frac{F}{p_{l}}\left(D^{2}-a^{2}\right)\right] Z=\frac{i k_{x} \mu_{e} H d^{2}}{4 \pi \rho_{0} \mathrm{v}} X} \\
& \left(D^{2}-a^{2}-p_{2} \sigma\right) K=-\frac{i k_{x} H d^{2}}{\eta \in} W+\frac{c_{i} k_{x} H d^{2}}{4 \pi N_{e} \eta} X \\
& \left(D^{2}-a^{2}-p_{2} \sigma\right) X=-\left(\frac{i k_{x} H d^{2}}{\eta \in}\right) Z-\frac{c_{i} k_{x} H}{4 \pi N_{e} \eta}\left(D^{2}-a^{2}\right) K, \\
& \left(D^{2}-a^{2}-E p_{1}\right) \Theta=-\left(\frac{g d^{2}}{k c_{p}}\right) W(G-1) .
\end{aligned}
$$

We now consider the case where both the boundaries are free as well as perfect conductors of heat. Since both the boundaries are maintained at constant temperature, the perturbations in the temperature are zero at the boundaries, therefore, the appropriate boundary conditions are

$$
\begin{aligned}
& W=D^{2} W=0, \quad D Z=0, \quad \Theta=0 \quad \text { at } \quad z=0 \quad \text { and } \quad 1, \\
& W=W_{0} \sin \pi z
\end{aligned}
$$

Eliminating $\Theta, X, Z$ and $K$ between Eqs (3.2)-(3.6), using Eqs (3.7) and substituting $W=W_{0} \sin \pi z$, we have

$$
\begin{aligned}
& \frac{G}{G-1}\left(\frac{1+x}{x}\right)\left[\frac{i \sigma_{1}}{\epsilon}+\frac{1}{P}+\frac{\pi^{2} F(1+x)}{P}\right]\left(1+x+i E p_{1} \sigma_{1}\right)+ \\
R_{1}= & \frac{+Q_{1} \cos ^{2} \theta(1+x)\left[1+x+i E p_{1} \sigma_{1}\right]\left\{\frac{i \sigma_{1}}{\epsilon}+\frac{1}{P}+\frac{\pi^{2} F(1+x)}{P}\left(1+x+i p_{2} \sigma_{1}\right)+Q_{1} x \cos ^{2} \theta\right\}}{\left[\frac{i \sigma_{1}}{\epsilon}+\frac{1}{P}+\frac{\pi^{2} F(1+x)}{P}\right]\left[1+x+i p_{2} \sigma_{1}\right]^{2}+Q_{1} x \cos ^{2} \theta\left[1+x+i p_{2} \sigma_{1}\right]+M x \cos ^{2} \theta(1+x)}, \\
& \times\left[\frac{i \sigma_{1}}{\epsilon}+\frac{1}{P}+\frac{\pi^{2} F(1+x)}{P}\right]
\end{aligned}
$$




$$
\begin{aligned}
& R_{l}=\frac{g \alpha \beta d^{4}}{v k \pi^{4}}, \quad Q_{1}=\frac{\mu_{e} H^{2} d^{2}}{4 \pi \rho_{0} \cup \eta \in \pi^{2}}, \quad M=\left(\frac{c H}{4 \pi N_{e} \eta}\right)^{2}, \quad x=\frac{a^{2}}{\pi^{2}}, \\
& i \sigma_{l}=\frac{\sigma}{\pi^{2}}, \quad P=\pi^{2} p_{l}, \quad k_{x}=k \cos \theta .
\end{aligned}
$$

\section{Stationary convection}

When the instability sets in as stationary convection, on putting $\sigma=0$ in Eq.(3.9), reduces to

$$
R_{1}=\left(\frac{G}{G-1}\right)\left(\frac{1+x}{x}\right)\left[\frac{\left(1+\pi^{2} F(1+x)\right)(1+x)}{P}+\frac{Q_{1} x \cos ^{2} \theta\left\{\frac{\left(1+\pi^{2} F(1+x)\right)(1+x)}{P}+Q_{1} x \cos ^{2} \theta\right\}}{\left.\left(\frac{1+\pi^{2} F(1+x)}{P}\right)\left(1+x+M x \cos ^{2} \theta\right)+Q_{1} x \cos ^{2} \theta\right]}\right]
$$

The above relation expresses the modified Rayleigh number $R_{l}$ as the function of parameters $Q_{1}, F, P$ and $M$ and dimensionless wave number $x$. For the fixed values of $P, M, Q_{1}, F$, let the nondimensional number $G$ (accounting for compressibility effects) be kept as fixed, then we get

$$
\overline{R_{c}}=\left(\frac{G}{G-1}\right) R_{c}
$$

where $R_{c}$ and $\overline{R_{c}}$ denote, respectively, the critical Rayleigh numbers in the presence and absence of compressibility. Since the critical Rayleigh number is positive and finite, so $G>1$, have the effect of compressibility is to postpone the onset of thermal instability. The case $G<1$ and $G=1$ corresponds to the negative and infinite values of the Rayleigh number which are not relevant in the present study. Hence, compressibility has a stabilizing effect on thermal convection under consideration.

For the case of stationary convection, to study the effect of the magnetic field, Hall currents, medium permeability and couple-stress, we examine the nature of $\frac{d R_{1}}{d Q_{1}}, \frac{d R_{1}}{d M}, \frac{d R_{1}}{d P}$ and $\frac{d R_{1}}{d F}$ analytically. From Eq.(4.1), we have

$$
\begin{aligned}
& \frac{d R_{1}}{d Q_{1}}=\left(\frac{G}{G-1}\right)\left(\frac{1+x}{x}\right) x \cos ^{2} \theta\left[\frac{\left(\frac{1+\pi^{2} F(1+x)}{P}\right)^{2}\left(1+x+M x \cos ^{2} \theta\right)(1+x)}{\left[\frac{1+\pi^{2} F(1+x)}{P}\left(1+x+M x \cos ^{2} \theta\right)+Q_{1} x \cos ^{2} \theta\right]^{2}}+\right. \\
& \left.+\frac{\left.2 Q_{1} \cos ^{2} \theta\left(\frac{1+\pi^{2} F(1+x)}{P}\right)\left(1+x+M x \cos ^{2} \theta\right)+Q_{1}^{2} x \cos ^{4} \theta\right]}{\left[\frac{1+\pi^{2} F(1+x)}{P}\left(1+x+M x \cos ^{2} \theta\right)+Q_{1} x \cos ^{2} \theta\right]^{2}}\right],
\end{aligned}
$$


which is positive, therefore magnetic field has postpone the onset of thermal convection in a compressible couple-stress fluids through porous medium.

$$
\frac{d R_{1}}{d M}=-\left(\frac{G}{G-1}\right) Q_{1} x(1+x) \cos ^{4} \theta\left[\frac{\left\{\left(\frac{1+\pi^{2} F(1+x)}{P}\right)(1+x)+Q_{1} x \cos ^{2} \theta\right\}\left\{\frac{1+\pi^{2} F(1+x)}{P}\right\}}{\left[\frac{1+\pi^{2} F(1+x)}{P}\left(1+x+M x \cos ^{2} \theta\right)+Q_{1} x \cos ^{2} \theta\right]^{2}}\right],
$$

which is negative, therefore Hall currents hasten postpone the onset of convection in a compressible couplestress fluid through porous medium.

$$
\begin{aligned}
& \frac{d R_{1}}{d P}=-\left(\frac{G}{G-1}\right)\left(\frac{1+x}{x}\right)\left(\frac{1+\pi^{2} F(1+x)}{P^{2}}\right)\left[\frac{\frac{(1+x)}{P^{2}}\left(1+x+M x \cos ^{2} \theta\right)^{2}\left(1+\pi^{2} F(1+x)\right)^{2}}{\left[\frac{1+\pi^{2} F(1+x)}{P}\left(1+x+M x \cos ^{2} \theta\right)+Q_{1} x \cos ^{2} \theta\right]^{2}}+\right. \\
& \left.+\frac{2 Q_{1} x \cos ^{2} \theta(1+x)\left(1+x+M x \cos ^{2} \theta\right)\left(\frac{1+\pi^{2} F(1+x)}{P}\right)+Q_{1}\left(x \cos ^{2} \theta\right)^{2}\left(1+x-M x \cos ^{2} \theta\right)}{\left[\frac{1+\pi^{2} F(1+x)}{P}\left(1+x+M x \cos ^{2} \theta\right)+Q_{1} x \cos ^{2} \theta\right]^{2}}\right]
\end{aligned}
$$

which shows, that medium permeability hasten the onset of convection on couple-stress fluid in presence of Hall currents for all wave numbers $(1+x)>M x \cos ^{2} \theta$.

$$
\begin{aligned}
& \frac{d R_{1}}{d F}=\left(\frac{G}{G-1}\right) \frac{(1+x)^{2} \pi^{2}}{x P}\left[\frac{\frac{(1+x)}{P^{2}}\left(1+x+M x \cos ^{2} \theta\right)^{2}\left(1+\pi^{2} F(1+x)\right)^{2}}{\left[\frac{1+\pi^{2} F(1+x)}{P}\left(1+x+M x \cos ^{2} \theta\right)+Q_{1} x \cos ^{2} \theta\right]^{2}}+\right. \\
& \left.+\frac{Q_{1}\left(x \cos ^{2} \theta\right)^{2}\left(1+x-M x \cos ^{2} \theta\right)+\frac{2 Q_{1} x \cos ^{2} \theta(1+x)\left(1+x+M x \cos ^{2} \theta\right)}{P}\left\{1+\pi^{2} F(1+x)\right\}}{\left[\frac{1+\pi^{2} F(1+x)}{P}\left(1+x+M x \cos ^{2} \theta\right)+Q_{1} x \cos ^{2} \theta\right]^{2}}\right],
\end{aligned}
$$

which shows that the couple-stress has postponed the onset of convection on the thermal instability of compressible couple-stress fluid through porous medium in hydromagnetics in the presence of Hall currents for all wave numbers $(1+x)>M x \cos ^{2} \theta$. It is to be noted that in the absence of Hall currents, Eq.(4.6) provides

$$
\frac{d R_{1}}{d F}=-\left(\frac{G}{G-1}\right) \frac{\pi^{2}}{x P}(1+x)^{3},
$$

which clearly shows that couple-stress hastens or postpones the onset of convection. Thus couple-stress has a dual character, in the absence of Hall currents it has postponed the onset of convection while in their presence, it may hasten the onset of convection. 


\section{Stability of the system and oscillatory modes}

Multiplying Eq.(3.2) by $W^{*}$ (the complex conjugate of $W$ ), integrating over the range of $z$ and making use of Eqs (3.3)-(3.6) together with boundary conditions (3.7), we get

$$
\begin{aligned}
& F I_{1}+\left(1+P_{l} \frac{\sigma}{\epsilon}\right) I_{2}-\frac{g \alpha K a^{2}}{v \beta}\left(\frac{G}{G-1}\right) P_{l}\left[I_{3}+E p_{1} \sigma^{*} I_{4}\right]+\frac{\mu_{e} \in \eta}{4 \pi \rho_{0} \mathrm{v}} P_{l}\left[I_{5}+p_{2} \sigma^{*} I_{6}\right]+ \\
& +\frac{\mu_{e} \in \eta d^{2}}{4 \pi \rho_{0} \mathrm{v}} P_{l}\left[I_{9}+p_{2} \sigma I_{10}\right]+d^{2}\left[\left(1+P_{l} \frac{\sigma^{*}}{\epsilon}\right) I_{8}+F I_{7}\right]=0
\end{aligned}
$$

where

$$
\left.\begin{array}{c}
I_{1}=\int_{0}^{1}\left(\left|D^{2} W\right|^{2}+2 a^{2}|D W|^{2}+a^{4}|W|^{2}\right) d z, \\
I_{2}=\int_{0}^{1}\left(|D W|^{2}+a^{2}|W|^{2}\right) d z, \\
I_{3}=\int_{0}^{1}\left(|D \Theta|^{2}+a^{2}|\Theta|^{2}\right) d z, \\
I_{5}=\int_{0}^{1}\left(\left|D^{2} K\right|^{2}+2 a^{2}|D K|^{2}+a^{4}|K|^{2}\right) d z, \\
I_{6}=\int_{0}^{1}\left(|\Theta|^{2}\right) d z, \\
I_{7}=\int_{0}^{1}\left(|D Z|^{2}+a^{2}|Z|^{2}\right) d z, \\
I_{8}=\int_{0}^{1}\left(|Z|^{2}\right) d z, \\
I_{9}=\int_{0}^{1}\left(|D X|^{2}+a^{2}|X|^{2}\right) d z, \\
I_{10}=\int_{0}^{1}\left(|X|^{2}\right) d z .
\end{array}\right\}
$$

All the integrals $I_{1}-I_{10}$ are positive definite. Putting $\sigma=\sigma_{r}+i \sigma_{i}$ in Eq.(5.1) and then equating the real and imaginary parts, we get 


$$
\begin{aligned}
& \sigma_{r}\left[\frac{I_{2}}{\epsilon}-\frac{g \alpha G}{G-1} \frac{k a^{2}}{\mathrm{v} \beta} E p_{1} I_{4}+\frac{\mu_{e} \in \eta}{4 \pi \rho_{0} \mathrm{v}} p_{2}\left(I_{6}+d^{2} I_{10}\right)+\frac{d^{2}}{\epsilon} I_{8}\right]= \\
& =-\left[\frac{F}{P_{l}} I_{1}+\frac{1}{P_{l}} I_{2}-\frac{G g \alpha k a^{2}}{(G-1) \cup \beta} I_{3}+\frac{\mu_{e} \in \eta}{4 \pi \rho_{0} \mathrm{v}}\left(I_{5}+d^{2} I_{9}\right)+\frac{d^{2}}{P_{l}}\left(I_{8}+F I_{7}\right)\right],
\end{aligned}
$$

and

$$
i \sigma_{i}\left[\frac{I_{2}}{\epsilon}+\frac{g \alpha k a^{2}}{v \beta} E p_{1} I_{4}-\frac{\mu_{e} \in \eta}{4 \pi \rho_{0} \mathrm{v}} p_{2}\left(I_{6}-d^{2} I_{10}\right)-\frac{d^{2}}{\epsilon} I_{8}\right]=0
$$

It may be inferred from Eq.(5.3) that $\sigma_{r}$ may be positive or negative which means that the system may be unstable or stable while Eq.(5.4) predicts that $\sigma_{i}=0$ necessarily because all the terms in the brackets are positive definite, which implies that oscillatory modes are not allowed in the system.

\section{Conclusion}

In the present paper we have investigated the effect of Hall currents on an electrically conducting couple-stress fluid layer heated from below in the presence of a horizontal magnetic field saturated in a porous medium. The dispersion relation governing the effects of Hall currents, horizontal magnetic field, porous medium, compressibility and couple-stress is derived. The main results are as follows:

(i): Compressibility has a stabilizing effect on the system from Eq.(4.2).

(ii): The magnetic field and couple-stress parameter has postponed the onset of convection in the presence of Hall current effect, from Eqs (4.3) and (4.6). Therefore the magnetic field and couple-stress have a stabilizing effect on the system.

(iii): For the case of stationary convection, Hall currents, and medium permeability hasten the onset of convection, from Eqs (4.4) and (4.5). Therefore Hall currents and medium permeability have a destabilizing effect on the system.

(iv): In the absence of Hall currents, the couple-stress hastens or postpones the onset of convection, from Eq.(4.7).

(v): Oscillatory modes are not allowed in the system and the principle of exchange of stabilities is satisfied in the present problem.

\section{Nomenclature}

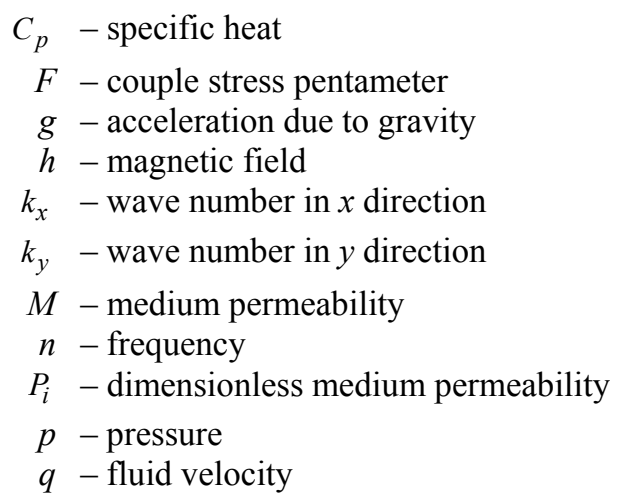




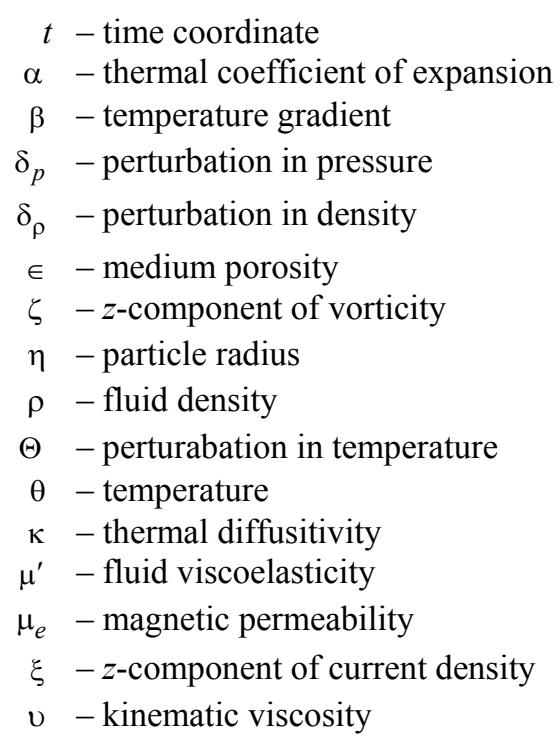

\section{References}

[1] Chandrasekhar S. (1961): Hydrodynamic and Hydromagnetic Stability. - Oxford: Clarendon Press.

[2] Stokes V.K. (1966): Couple-stress in fluids. - Phys. Fluids, vol.9, No.9, pp.1709-1715.

[3] Stokes V.K. (1984): Theories of Fluids with Microstructure. - New York: Springer-Verleg.

[4] Walicki E. and Walicka A. (1999): Inertia effect in the squeeze film of a couple-stress fluids in biological bearings. - Int. J. Appl. Mech. Engg., vol.4, No.2, pp.363-373.

[5] Sunil, Sharma R.C. and Chandel R.S. (2004): Effect of suspended particles on couple-stress fluid heated and soluted from below in porous medium. - J. Porous Media, vol.7, No.1, pp.9-18.

[6] Sharma R.C. and Sharma M. (2004): Effect of suspended particles on couple-stress fluid heated from below in the presence of rotation and magnetic field. - Indian J. Pure and Appl. Math., vol.35, No.8, pp.973-989.

[7] Chandra K. (1938): Instability of fluids heated from below. - Proc. Roy. Soc., A164, pp.231-242.

[8] Spiegal E.A. and Veronis G. (1960): On the boussinesq approximation for a compressible fluid. - Astrophys. J., vol.131, No.2, pp.442-444.

[9] Sharma R.C., Sunil, Sharma Y.D. and Chandel R.S. (2002): On couple-stress fluid permeated with suspended particles heated from below. - Arch. Mech., vol.54, No.3, pp.287-298.

[10] Malashetty M.S. and Kollur P. (2011): The onset of double-diffusive convection in a couple-stress fluid saturated anisotropic porous layer. - Transport in Porous Media, vol.86, pp.435-459.

[11] Singh M. (2011): Hall current effects on thermosolutal instability in a viscoelastic fluid flowing in a porous medium. - Int. J. Applied Mechanics and Engineering, vol.16, No.1, pp.69-82.

[12] Jaimala B., Jawla V. and Kumar V. (2013): Thermal convection in a couple-stress fluid in the presence of horizontal magnetic field with Hall currents. - AAM, Int. J., vol.8, No.1, pp.161-177.

[13] Singh V. and Dixit S. (2012): Hall effect on thermal instability of compressible couple-stress fluid in presence of suspended particles. - Int. J. Mathematical Arch., vol.3, No.4, pp.1701-1711.

[14] Singh M. and Kumar P. (2011): Magneto and rotatory thermosolutal convection in couple-stress fluid in porous medium. - Journal of Porous Media, vol.14, No.7, pp.637.

[15] Singh M. and Mehta C.B. (2013): On compressible couple-stress fluid heated and soluted from below in porous medium in presence of rotation and magnetic field. - Journal of Mechanical Engineering and Technology, vol.1, No.1, pp.21. 
[16] Singh M. and Kumar P. (2012): Thermosolutal convection in a couple-stress fluid in uniform magnetic field. - Z. Naturforsch, vol.67a, pp.275-281.

[17] Krishna M.V. and Neeraja E. (2013): Hall effect on MHD flow of a couple-stress fluid in a parallel plate channel bounded by a porous bed on the lower half in presence of inclined magnetic field. - Int. J. Advanced Technology and Engineering Research, vol.3, No.3, pp.103.

[18] Rudraiah N., Shankar B.M. and Chiu N.G. (2011): Electro hydrodynamic stability of couple-stress fluid flow in a channel occupied by a porous medium. - Special Topics Rev. Porous Media., vol.2, pp.11-22.

Received: February 20, 2015

Revised: December 17, 2015 